\title{
Measurement of heat transfer coefficient using termoanemometry methods
}

\author{
P. Dančová ${ }^{1,2, a}$, P Sitek $^{1}, T$. Vít ${ }^{1}$ \\ ${ }^{1}$ Technical university of Liberec, Dept. of Power Engineering Equipment, Studentská 2, 46117 Liberec 1, \\ Czech Republic \\ ${ }^{2}$ Institute of Thermomechanics, CAS, v.v.i., Dolejškova 5, 18000 Praha 8, Czech Republic
}

\begin{abstract}
This work deals with a measurement of heat transfer from a heated flat plate on which a synthetic jet impacts perpendicularly. Measurement of a heat transfer coefficient (HTC) is carried out using the hot wire anemometry method with glue film probe Dantec 55M47. The paper brings also results of velocity profiles measurements and turbulence intensity calculations.
\end{abstract}

\section{Introduction}

The aim of this work is to measure the heat transfer coefficient in air as a working fluid, when synthetic jet (SJ) impacts perpendicularly on a heated flat plane.

The inspiration for this paper was work of Sholten and Murray $[1,2]$. The technique described in their work was applied to the heated tube placed in a wind tunnel perpendicularly to the air flow. The method was based on the difference of heat generated in the film probe at non-zero flow ( $\mathrm{SJ}$ is switched on), and the heat generated in the film probe at zero flow of SJ.

The problem of heat/mass transfer was also investigated in [3] where the mass transfer was measured using naphthalene technique and the heat transfer on the wall was calculated with the heat/mass transfer analogy.

A synthetic jet (SJ) is generated by the periodic motion of an actuator oscillating membrane. A SJ is synthesized by the interactions within a train of vortex rings or counter-rotating vortex pairs in axissymmetric or two-dimensional geometry; see Smith and Glezer [4]. Vortex rings are formed at the lip of the orifice (see figure 1). These rings move from the actuator orifice with a velocity, which must be high enough to prevent interaction with suction in the orifice. It was observed that an SJ far enough from the orifice has character of a conventional steady jet. This is caused by both the development and the dissipation of vortexes. One of the main advantages of a $\mathrm{SJ}$ is that the time-mean mass flux of the oscillatory flow in the orifice is zero; hence the other common expression is a zero-net-massflux (ZNMF) jet. A ZNMF eliminates the requirement of a blower and piping for the fluid inlet. Though the SJ actuator works with a ZNMF at the orifice, the momentum of the resultant SJ at a specific distance from the lip in axis is non-zero [4].

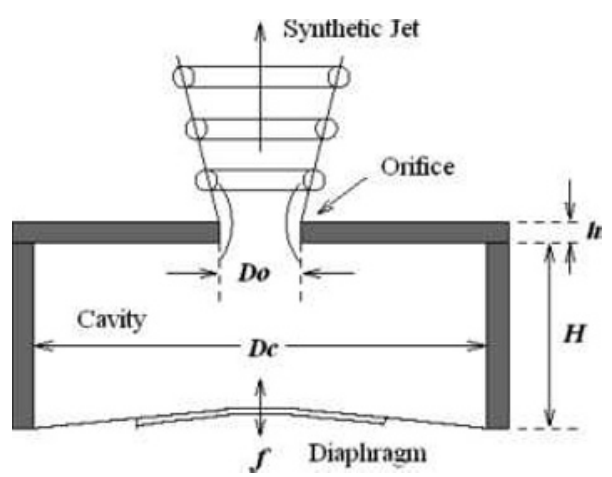

Figure 1. Basic principle of SJ, [4].

The equipment for a SJ can have various designs, but the main mechanism and principle are generally the same. Figure 1 shows the simplest setting: There is an orifice at one end of the actuator, whereby the fluid is periodically sucked in / exhausted from the actuator cavity. The pulsation generator of the fluid can work on the principle of loudspeaker, piezo crystal, electromagnet, piston, or other device. It is necessary to choose an optimal type and construction of actuator in relation to the supposed

\footnotetext{
${ }^{\mathrm{a}}$ Corresponding author: petra.dancova@tul.cz
} 
working frequency range, working temperature, kind of working medium, and required load of the unit.

A SJ is characterized by several independent parameters, [4]. When considering the plug flow model (one-dimensional piston - like flow in the actuator orifice), the main parameters of a $\mathrm{SJ}$ are: the actuator orifice diameter $D$, the mean time orifice velocity $U_{0}$

$$
U_{0}=f \int_{0}^{\frac{T}{2}} u_{0}(t) d t
$$

where $T$ means the time period, i.e. $T=1 / f$ and $f$ means the frequency, $T_{\mathrm{E}}$ means extrusion time ( $T_{\mathrm{E}}=T / 2$ for the sinusoidal waveform), $u_{0}(t)$ means the periodical axial orifice velocity. For the sinusoidal waveform $u_{0}(t)=U_{\max } \sin (2 \pi f t)$.

Next important parameters are the "stroke length" $L_{0}$

$$
L_{0}=U_{0} . T
$$

and a Reynolds and Strouhal numbers

$$
R e=U_{0} D / v, S t=D / \pi L_{0},
$$

where $v\left(\mathrm{~m}^{2} / \mathrm{s}\right)$ is kinematic viscosity.

\section{Experimental setup}

Experimental setup was made from a SJ actuator and a heated flat plate. The plate was made from aluminum of dimensions $(120 \times 120) \mathrm{mm}$ and was put into a wooden frame and firmly fixed. The SJ actuator was placed on a traverser, which enabled movement in all directions of Cartesian coordinate system.

The SJ actuator consisted from a sealed cavity with an orifice (diameter $D=10 \mathrm{~mm}$ ) and two loudspeakers TVM ARN-100-10/4 in position visible in figure 2. This design was taken from [5].

Both loudspeakers were fed with sinusoidal signal from signal generator Tektronik AFG 2102 and amplified from amplifier Omnitronic MPZ180.

For heat transfer experiments, a glue film probe, Dantec 55M47, is fixed on the heated aluminum plate and is connected into anemometer Dantec 90C10 in mode of constant temperature.

For velocity profile measurement, hot wire probe Dantec 55P11 was used. The SJ actuator was fixed firmly and the probe was moved with traverser.

\section{Experimental results}

During the experiments, two signals were recorded - signal from anemometer and TTL signal. TTL signal was changed between $3.4 \mathrm{~V}$ and $0.1 \mathrm{~V}$, where value of $3.4 \mathrm{~V}$ corresponds to the phase of exhaust from SJ actuator and value $0.1 \mathrm{~V}$ to the phase of suction into SJ actuator. According to TTL signal, the measured signal from anemometer was divided for phase averaging.

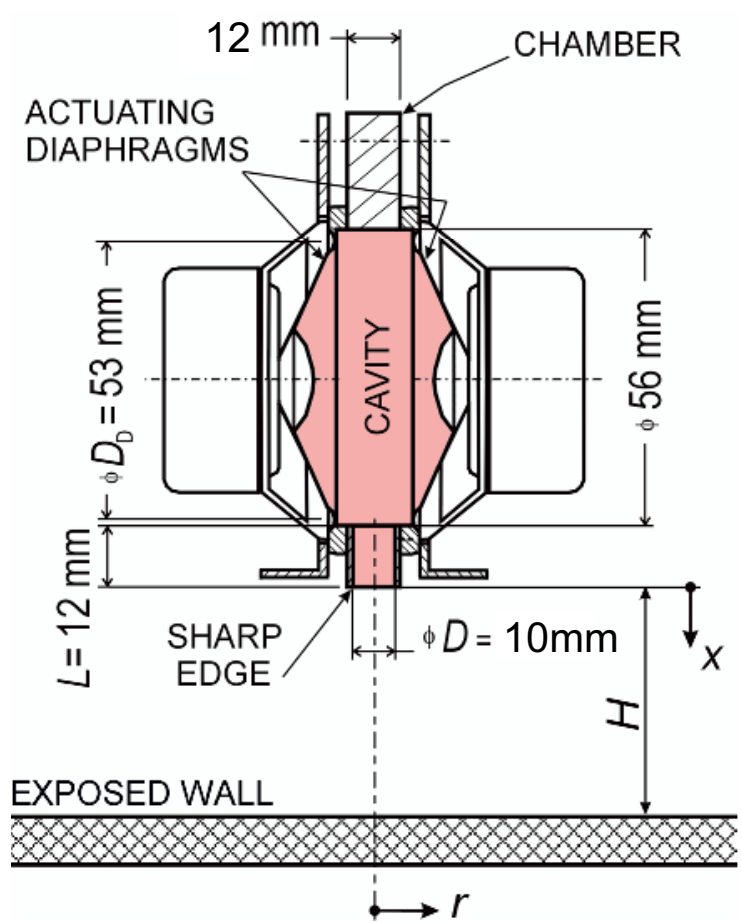

Figure 2. Design of SJ actuator, Z. Trávníček et al. [5].

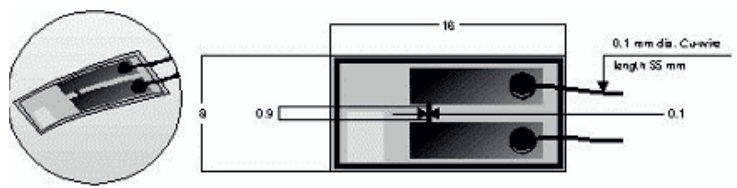

Figure 3. Glue film probe Dantec 55M47.

\subsection{Experimental investigation and calculation of heat transfer coefficient}

To determine the heat transfer coefficient, it was necessary to measure the voltage on the Wheatston'n bridge without flow of SJ firstly, then to measure the voltage with switched SJ. From these two values, from material constants of the probe and from set resistances of the probe, the heat released to the surroundings by convection can be calculated as

$$
Q_{\text {diss }}=\left(V_{\text {forced }}^{2}-V_{0}^{2}\right) \frac{R_{\text {senzor }}}{\left(R_{\mathrm{arm}}+R_{\mathrm{top}}\right)^{2}},
$$

where $V_{\text {forced }}$ was the voltage on the bridge with flow of the SJ, $V_{0}$ the voltage at the bridge without the $\mathrm{SJ}, R_{\text {senzor }}$ the constant resistance dependent on the probe overheat ratio, $R_{\text {arm }}$ the resistance of cable and probe holder, $R_{\text {top }}$ the resistance of the Weatston's bridge branch where the probe was also connected.

The resulting heat transfer coefficient (HTC) $\alpha$ was obtained when $Q_{\text {diss }}$ was divided by difference between temperature of the probe and of the 
flowing medium and by the effective area of the probe

$$
\alpha=\frac{Q_{\text {diss }}}{1,5 A\left(T_{\mathrm{w}}-T_{\mathrm{a}}\right)} .
$$

\subsubsection{Heat transfer coefficient measurement}

Experiments were performed for three different overheat ratios at distances of 3.5, 5, 10 and 20 diameters from the actuator orifice. SJ actuator was fed with the sinusoidal signal with frequency of $30 \mathrm{~Hz}$ with an output of $1.6 \mathrm{~W}$. Frequency of $30 \mathrm{~Hz}$ was not the actuator eigen frequency, but it was chosen because of slow capturing speed of the probe. Sampling frequency was set at $3 \mathrm{kHz}$ and the number of samples was set to 4096 .

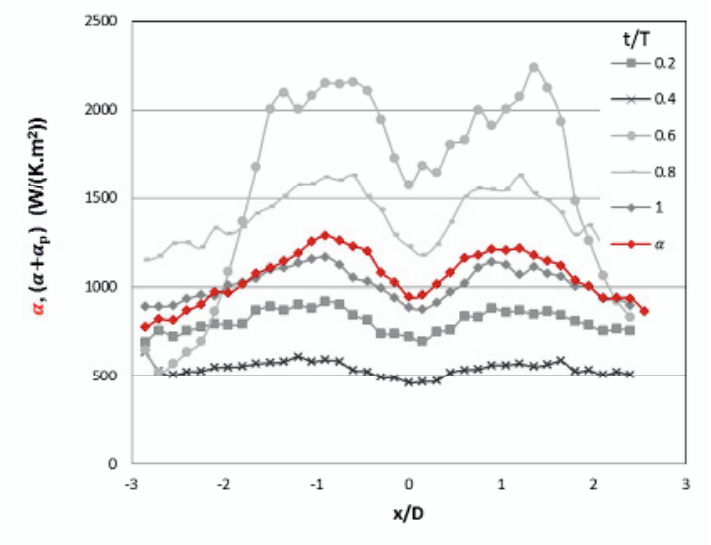

Figure 4. Phase averaged HTC and time mean value of HTC measured in plane $x z$ at distance $y / D=5$

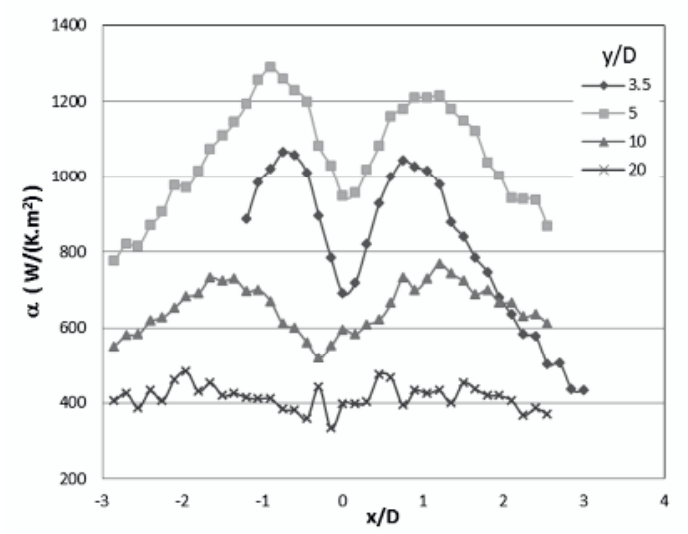

Figure 5: Time mean values of HTC in plane $x z$.

Figure 4 shows HTC as the phase-averaged and the time-mean values in distance $y / D=5$ from the orifice. The highest values were reached during the phase of extrusion from the actuator, i.e. $t / T=(0.6-$ 1). On figure 5 the time mean values of HTC in different distances from the orifice are visible. In the vicinity of the SJ actuator, the impact of vortex rings on a flat plate can be observed, thereby a significant heat transfer occurs. With increasing distance, the significant difference between the vortex ring center and the ring itself decreases. At distance $y / D=20$, the SJ has character of a free jet (also visible on figure 6), therefore the HTC differences are very low.

\subsection{Measuring of velocity profiles}

For velocity experiments, the actuator was fed with sinusoidal signal with frequency of $70 \mathrm{~Hz}$, which was the actuator eigen frequency, with output of 1.6 W. Sampling frequency and number of samples was $64 \mathrm{kHz}$ and 32768, respectively.

Figure 6 demonstrates the dependence of the phase-averaged velocity in different instants of the period and the time-mean velocity on the distance from the actuator orifice. There was a visible decrease of velocity when the distance increased along $y$ direction. At the distance from the orifice $y / D=15$, the flow oscillation practically vanished and the flow can be considered as a steady jet flow. Further downstream from the orifice, the vortex structure also vanished owing to dissipative processes; thereby the velocity further was decreasing.

Figure 7 shows the time mean velocity magnitude profiles, which were measured at $y / D=$ 1,2 , and 4 .

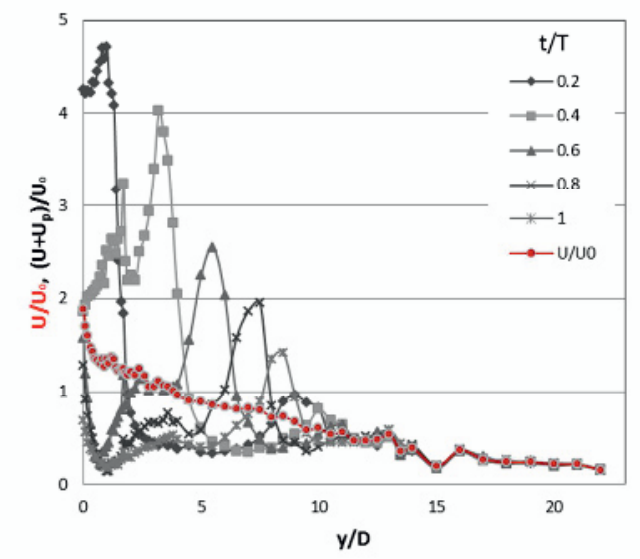

Figure 6. Dependence of the phase-averaged velocity magnitude at different instances in the period and of the time-mean velocity on the distance from the actuator orifice.

\subsection{Turbulence intensity calculation}

The turbulence intensity at a particular time was calculated from values from velocity experiments in the $x z$ plan performed at distances $y / D=1,2$, and 4 as

$$
T_{\mathrm{i}}(t / T)=\frac{[e(t / T)]_{\mathrm{rms}}}{\bar{E}+e_{\mathrm{p}}(t / T)},
$$

where $\bar{E}$ is the time mean component of voltage measured on a probe, $e_{\mathrm{p}}(t / T)$ is the periodic part, and $e(t / T)_{\text {rms }}$ is the standard deviation in the phase $t / T$. 


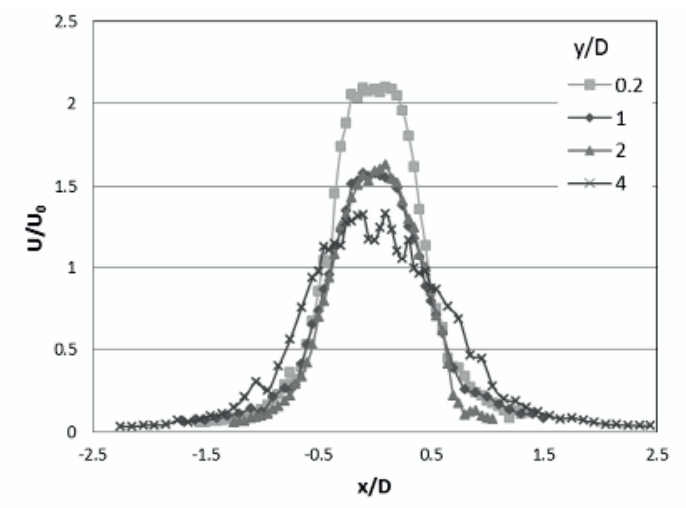

Figure 7. Time mean velocity profiles at distances $y / D=1,2$, and 4 , measured in plane $x z$.

As it is visible from figure 8, the highest values of turbulence intensity are in places of vortex rings and correspond well with shapes of HTC in figure 5 .

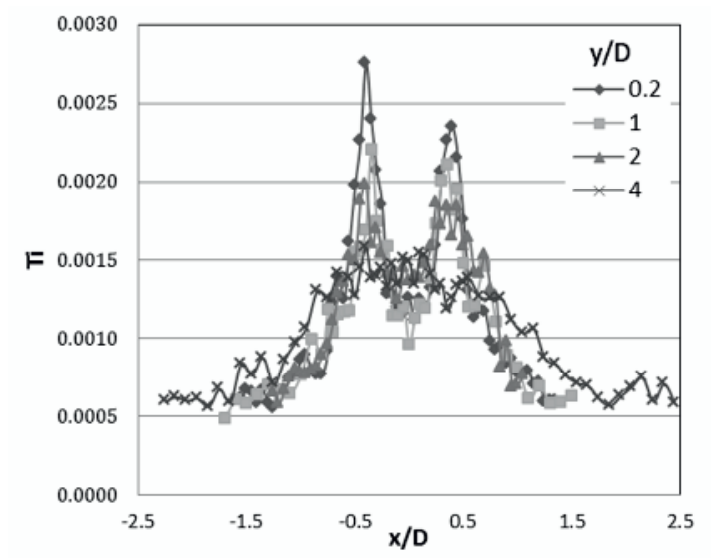

Figure 8. Time mean values of turbulence intensity in plane $\mathrm{xz}$ at different distances from the $\mathrm{SJ}$ actuator orifice.

\section{Conclusion}

In this work, the heat transfer coefficient on the heated wall, which was cooled by impacted SJ, was measured. The SJ velocity profiles were also measured along the axis of the SJ actuator orifice and in the plane $x z$, which was perpendicular to the axis of the flow. The turbulence intensity was calculated from values of the velocity measurement in plane $x z$. The measurement was carried out at two different frequencies, one for HTC and the second one for velocities experiments. The value of HTC was obtained from the ratio of convective heat, temperature difference and the effective probe area.

HTC was measured in the range of (100$5000) \mathrm{W} /\left(\mathrm{K} . \mathrm{m}^{2}\right)$ and the highest value was identified at the distance of 5 diameters from the SJ actuator orifice. During the velocity experiments in vicinity of the SJ actuator orifice, the mean time orifice velocity was estimated as $4.08 \mathrm{~m} / \mathrm{s}$. Also it was demonstrated that from the distance of 15 diameters, the SJ lost character of the pulsating flow.

\section{References}

1. J.W. Scholten, D.B. Murray, Transactions of the ASME 118, 982 (1996)

2. J.W. Scholten, D.B. Murray, Int. J. Heat Mass Transfer 41 (10), 1139 (1998)

3. Z. Trávníček, V. Tesař, Int. J. Heat Mass Transfer 46, 3291 (2003)

4. B.L. Smith, A. Glezer: Phys. Fluids 10, 2281 (1998)

5. Z. Trávníček, J. Vogel, T. Vít, F. Maršík, $4^{\text {th }}$ International Conference on Heat Transfer, Fluid Mechanics, and Thermodynamics (HEFAT 2005), paper No. ZT4 (2005)

6. Z. Trávníček, V. Tesař, Int. J. of Heat and Mass Transfer 47, 2329 (2004)

7. http://www-g.eng.cam.ac.uk/whittle/currentresearch/hph/hot-film/hot-film.html (cited 15/04/2013)

8. P. Sitek, Measuring of heat transfer coefficient using hot wire anemometry, Bachelor thesis - in Czech (2006)

9. P. Dančová, Experimental investigation of synthetic jet in a laminar channel flow, Dissertation thesis (2012)

10. K. Kaduchová, R. Lenhard, S. Gavlas, J. Jandačka, EPJ Web of Conferences 45, 01125 (2013)

\section{Acknowledgement}

This work was supported by ESF operational programme "Education for Competitivness" in the Czech Republic in the framework of project „Support of engeneering of excellent research and development teams at the Technical University of Liberec" No. CZ.1.07/2.3.00/30.0065.

We also thank to the project SGS 28000. 